\title{
Influence of free surface curvature of a liquid layer on the critical Marangoni convection
}

\author{
Z.M. Tang *, K. Li, W.R. Hu \\ National Microgravity Laboratory, Institute of Mechanics, Chinese Academy of Sciences, Beijing 100080, China
}

\section{A R T I C L E I N F O}

\section{Article history:}

Received 30 October 2007

Received in revised form 24 March 2008

Available online 20 May 2008

\section{Keywords:}

Liquid layer

Large Prandtl number

Pearson instability

Thermocapillary flow

Marangoni flow

\begin{abstract}
A B S T R A C T
The Pearson instability was suggested to discuss the onset of Marangoni convection in a liquid layer of large Prandtl number under an applied temperature difference perpendicular to the free surface in the microgravity environment. In this case, the temperature distribution on the curved free surface is nonuniform, and the thermocapillary convection is induced and coupled with the Marangoni convection. In the present paper the effect of volume ratio of the liquid layer on the critical Marangoni convection and the corresponding spatial variation of the convection structure in zero-gravity condition were numerically investigated by two-dimensional model.
\end{abstract}

(c) 2008 Elsevier Ltd. All rights reserved.

\section{Introduction}

Pearson's theoretical analysis, by means of small-disturbance theory, indicated that Marangoni convection can be induced in a fluid layer of infinite extension heated from the liquid-solid boundary at the bottom, when the Marangoni number is larger than a critical value $M a_{c}$ [1]. This phenomenon suggested a new instability mechanism in a fluid system, which is different from Rayleigh instability [2], and it plays an important role in the engineering applications. Many works have been devoted to the investigation of Marangoni convection in liquid layers. Most works assumed that the free surface is planar and nondeformable, and focused on the structure of Marangoni convection in liquid layers with different aspect ratios [3,4]. These works analyzed the influence of lateral slippery boundary condition and indicated that the time-periodic convection exists in some cases. Dijkstra et al. investigated the surface tension driven cellular patterns in three-dimensional boxes. Their results were summarized as a map in the $x$ - and $y$-aspect ratio plane of the preferred modes, and discussed the effect of boundary conditions [5-7]. Surface tension plays an important role in the micro-size fluid dynamics, for example in thermal management of electronic devices, food processing and chemical engineering. The Marangoni convection and heat transfer in thin liquid films heated on walls with topography and with mobile gas-liquid interface was investigated [8]. If the substrate has a structure

\footnotetext{
* Corresponding author. Tel.: +86 10 82544119; fax: +86 1082544096

E-mail address: zmtang@imech.ac.cn (Z.M. Tang).
}

on its upper surface, the convection prevails for any temperature difference. It is found that the substrate structure modifies the flow patterns and affects the heat and mass transfer in the liquid film. The investigation on thermocapillary flows under an inclined temperature gradient was conducted by Oleg and Alexander [9]. The results brought to light the importance of the horizontal temperature gradient on the formation and the development of convective regimes in a plane liquid layer heated from liquid/solid boundary at the bottom.

The geometric simplification of planar and non-deformable free surface adopted in the aforementioned investigations is not real in practice. The free surface of a liquid layer in the microgravity environment is curved in general due to weaker static pressure than that on the ground and stronger surface tension. Thus, even if the applied temperature gradient is perpendicular strictly to the liquid layer, there is a temperature difference along the curve free surface, which means the thermocapillary convection and the Marangoni convection coexist and interact with each other in the microgravity environment.

In the present paper, we investigate the coupled thermocapillary convection and Marangoni convection in a fluid layer of finite extension with a curved free surface in the microgravity environment by using two-dimensional numerical simulation. The effect of volume ratio of the liquid layer on the critical Marangoni number is studied in detail. The physical model and mathematical formulation of the problem are given in the next section. In Section 3 we describe the numerical method and the code validation. The computed results are presented in Section 4. The last section is the discussion. 


\section{Physical model and mathematical formulation}

As shown in Fig. 1, a two-dimensional cavity is partially filled with 10 cst silicon oil $(P r=105.6)$. The liquid layer is surrounded by an ambient gas with temperature $T_{\mathrm{e}}\left(25^{\circ} \mathrm{C}\right)$. The heights of the liquid layer at both lateral sides are $h_{0}=3 \mathrm{~mm}$ and the length of the liquid layer is $l=55.5 \mathrm{~mm}$. By heating the bottom of the container up to a given value $T_{\mathrm{b}}$, a vertical temperature gradient is set up in the liquid layer. Heat is transferred from the liquid to the gas, and the heat transfer coefficient is $q$ at the free surface. Both side walls are assumed to be thermally isolated. The liquid is considered as incompressible with constant viscosity and thermal diffusivity, and Boussinesq approximation is adopted. The surface tension $\sigma$ on the free surface is a linear function of the temperature $T$, and $-\partial \sigma / \partial T$ is assumed to be constant. The free surface is described as $y=h(x)$. The height $h_{0}$ of liquid layer at both lateral sides, the temperature difference $\Delta T=T_{\mathrm{b}}-T_{\mathrm{e}}$, velocity $U_{\mathrm{r}}=|\partial \sigma / \partial T| \Delta T / \mu$ and pressure $\mu U_{\mathrm{r}} / h_{0}$ are used as the typical units to scale the length, temperature, velocity and pressure, where $\mu$ is dynamic viscosity.

Introducing the non-dimensional vorticity vector $\omega=\left(\omega_{\xi}, \omega_{\theta}, \omega_{\varsigma}\right)$ and stream function vector $\psi=\left(\psi_{\xi}, \psi_{\theta}, \psi_{\varsigma}\right)$, which are defined as

$\nabla \times \psi=V$

$\nabla \times \nabla \times \psi=\omega$.

The non-dimensional equations and boundary conditions can be expressed by using vorticity $\omega$ and stream function $\psi$. The mass conservation equation is satisfied automatically. The momentum conservation equation and energy conservation equation can be expressed respectively as Eqs. (3) and (4) as follows:

$$
\begin{aligned}
& \frac{\partial \omega}{\partial t}+V \cdot \nabla \omega-\omega \cdot \nabla V=\frac{1}{R e^{*}} \nabla^{2} \omega, \\
& \frac{\partial \Theta}{\partial t}+V \cdot \nabla \Theta=\frac{1}{M a^{*}} \nabla^{2} \Theta,
\end{aligned}
$$

where $\Theta=\frac{T}{\Delta T}$ is non-dimensional temperature.

For the two-dimensional problem, the boundary conditions are as follows:

$$
\begin{aligned}
& x=0 \text { and } x=\ell / h_{0}: \psi=0, \quad \omega=\frac{\partial^{2} \psi}{\partial x^{2}}=0, \quad \frac{\partial T}{\partial x}=0, \\
& y=0: \psi=0, \quad \frac{\partial \psi}{\partial x}=0, \quad T=T_{\mathrm{b}} .
\end{aligned}
$$

At the free surface $y=h(x) / h_{0}$ :
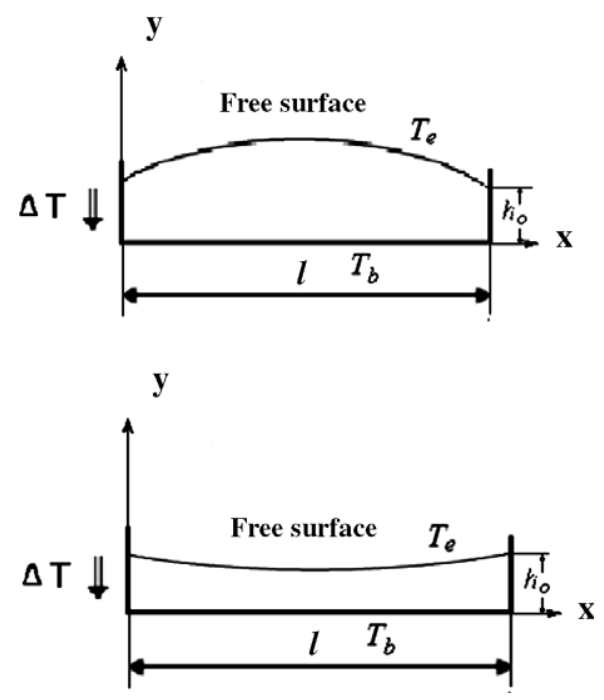

Fig. 1. A sketch of liquid layer with curved free surface.

$$
\begin{aligned}
& \psi=0, \\
& \omega=\frac{\left(1+h^{\prime 2}\right)}{\left(1-h^{\prime 2}\right)} \frac{\partial T}{\partial S}-\frac{4 h^{\prime}}{\left(1-h^{\prime 2}\right)}\left[\frac{\partial^{2} \psi}{\partial x \partial y}\right]+2 \frac{\partial^{2} \psi}{\partial y^{2}}, \\
& -\frac{\partial T}{\partial n}=L T,
\end{aligned}
$$

where $L=q h / \lambda, \lambda$ notes thermometric conductivity of the liquid and $q$ is the rate of heat loss per unit area from the upper surface. The vorticity at the free surface is determined from equilibrium of tangential stress (7), in which $S$ points the tangential direction. Eq. (8) means that the rate of heat supply to the surface from the liquid must equal to the rate of heat loss from the surface to its upper environment. $L$ depends on the efficiency of the process for transferring heat from the surface. The value of $q$ depends on the circumstances, and we shall retain it as a parameter in the subsequent calculation.

The following equilibrium of normal stress is used to calculate the shape of the free surface.

$$
\left(P-P_{\infty}\right)=\frac{2}{\left(1+h^{\prime 2}\right)}\left[h^{\prime 2} \frac{\partial U}{\partial x}-h^{\prime}\left(\frac{\partial W}{\partial x}+\frac{\partial U}{\partial y}\right)+\frac{\partial W}{\partial y}\right]-\frac{h^{\prime \prime}}{C a \cdot\left(1+h^{\prime 2}\right)^{3 / 2}},
$$

where $U$ and $W$ are, respectively the non-dimensional velocities in the directions $x$ and $y$, and the dynamic deformation of the free surface during heating process is negligible. The free surface at both the left and right boundaries locates at $y=1$, which means the method of controlling the height of the liquid layer at the interface of solid and liquid is adopted. The initial condition may be obtained from the static case without applied temperature difference and convection. The initial shapes of the free surfaces are different for the liquid layers with different volume ratio. The shape of the free surface and the volume of the liquid layer with a certain volume ratio are fixed in the calculating process.

\section{Numerical method and code validation}

Eqs. (1) and (4) with the initial and boundary conditions are solved by using the finite element method. The numbers of the cell are $121 \times 21$ in the directions $x, y$, respectively, and the calculated domain is divided in to 4800 triangular elements with 2541 nodes. The shape of the free surface is determined according to the volume ratio by using Eq. (9) with $U=0$, and $W=0$.

A hybrid finite element method of fractional steps has been used to solve the governing Eqs. (3) and (4) with boundaries (5)(8). In order to account the non-linear convection effect the characteristic procedure is used for convection operator, and the finite element method for diffusion calculation. The hybrid method of fractional steps was first suggested by Vanenko [10], and the hybrid finite element method of fractional steps was presented by $\mathrm{Wu}$ for numerical modeling of the pollution in aquatic environment in 1985. Stability analysis in detail and a two-dimensional sample for checking the divergence and precision were report in the paper $\mathrm{Wu}$ [11]. Since the method is a hybrid scheme of fractional steps on irregular triangular grids, which is stability, simplicity and flexibility in handling complex geometry, therefore, it is applied to simulate the thermocapillary-Marangoni convection of the liquid layer with different volume ratio in the present paper.

The present program is used to calculate the thermocapillary convection in a square container for comparing with the results obtained by Carpenter and Homsy. The results are shown in Table 1, where, $-\psi_{\min }$ is the minimum of stream function in the region, and $\omega_{\text {core }}$ is the vorticity at point $\left(\xi_{c}, \zeta_{c}\right)$. The non-dimensional velocity at $\xi=0.5$ and $\zeta=1$ is pointed by $u(0.5,1)$. Nusselt number is defined as $N u(\zeta)=\int_{0}^{1}\left(M a \frac{\partial \psi}{\partial \zeta} \Theta-\frac{\partial \Theta}{\partial \zeta}\right) \mathrm{d} \zeta$. The results obtained by the hybrid finite element method of fractional steps are coincident with those 
Table 1

Comparison of Carpenter's results [12] and present results: (A - results of Carpenter and Homsy, B - results of present paper)

\begin{tabular}{|c|c|c|c|c|c|c|c|c|c|}
\hline & Grid & $\operatorname{Re}$ & $-\psi_{\min }$ & $\omega_{\text {core }}$ & $\xi_{\mathrm{c}}$ & $\zeta_{\mathrm{c}}$ & $u(0.5,1)$ & $N u_{x=0}$ & $N u_{x=1}$ \\
\hline \multicolumn{10}{|c|}{$\operatorname{Pr}=1$} \\
\hline A & $64 \times 64$ & $1.0 \times 10^{4}$ & $3.23 \times 10^{-3}$ & $-6.97 \times 10^{-2}$ & 0.58 & 0.62 & $2.96 \times 10^{-2}$ & 4.36 & 4.40 \\
\hline B & $64 \times 64$ & $1.0 \times 10^{4}$ & $3.20 \times 10^{-3}$ & $-7.10 \times 10^{-2}$ & 0.58 & 0.63 & $2.95 \times 10^{-2}$ & 4.34 & 4.32 \\
\hline \multicolumn{10}{|c|}{$\operatorname{Pr}=10$} \\
\hline A & $64 \times 64$ & $1.0 \times 10^{3}$ & $2.60 \times 10^{-3}$ & $-10.0 \times 10^{-2}$ & - & - & $2.30 \times 10^{-2}$ & 3.79 & 3.83 \\
\hline B & $64 \times 64$ & $1.0 \times 10^{3}$ & $2.75 \times 10^{-3}$ & $-9.56 \times 10^{-2}$ & - & - & $2.47 \times 10^{-2}$ & 3.97 & 3.86 \\
\hline \multicolumn{10}{|c|}{$\operatorname{Pr}=30$} \\
\hline A & $64 \times 64$ & $0.25 \times 10^{3}$ & $2.18 \times 10^{-3}$ & $-9.96 \times 10^{-2}$ & - & - & $1.78 \times 10^{-2}$ & 3.34 & 3.37 \\
\hline B & $64 \times 64$ & $0.25 \times 10^{3}$ & $2.15 \times 10^{-3}$ & $-9.33 \times 10^{-2}$ & - & - & $1.74 \times 10^{-2}$ & 3.51 & 3.41 \\
\hline \multicolumn{10}{|c|}{$\operatorname{Pr}=50$} \\
\hline A & $74 \times 74$ & $0.20 \times 10^{3}$ & $1.96 \times 10^{-3}$ & $-9.07 \times 10^{-2}$ & - & - & $1.53 \times 10^{-2}$ & 3.59 & 3.57 \\
\hline B & $64 \times 64$ & $0.20 \times 10^{3}$ & $2.09 \times 10^{-3}$ & $-9.13 \times 10^{-2}$ & - & - & $1.74 \times 10^{-2}$ & 3.81 & 3.62 \\
\hline B & $74 \times 74$ & $0.20 \times 10^{3}$ & $2.12 \times 10^{-3}$ & $-9.03 \times 10^{-2}$ & - & - & $1.72 \times 10^{-2}$ & 3.84 & 3.64 \\
\hline
\end{tabular}

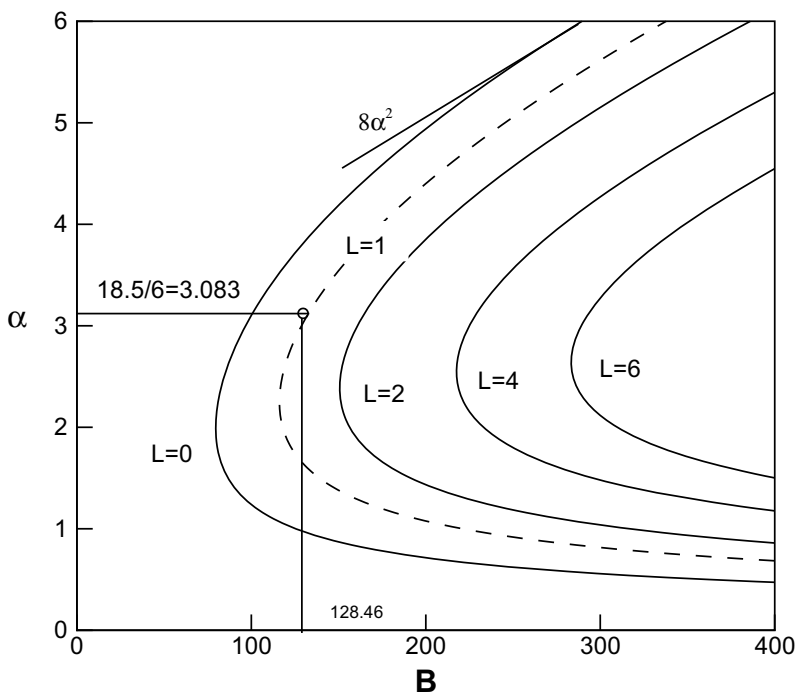

Fig. 2. The comparison with Pearson's results.

given by Carpenter. The similar results calculating by two kinds of different grids are given in Table 1 for the fluid of $\operatorname{Pr}=50$.

The present code is used to search the critical Marangoni number $M a_{\mathrm{c}}$ of a 10 cst silicon oil liquid layer with a planar free surface heated from below. The liquid layer is $3 \mathrm{~mm}$ in height, $55.5 \mathrm{~mm}$ in length, and the related aspect ratio is $l / h_{0}=18.5$. The parameter $L=1$ is adopted. The result gives the critical temperature difference $\Delta T_{\mathrm{c}}=1.93^{\circ} \mathrm{C}$, which corresponds $B=\frac{|\partial \sigma / \partial T| \beta h_{0}^{2}}{\rho v_{\mathrm{c}}}=128.46$. There is a convective flow upward at the central line of the liquid layer. The liquid layer includes 6 flow cells, therefore, the ratio of cell size to layer thickness, i.e. the dimensionless flow cell size, $\alpha=18.5 /$ $6=3.083$. Fig. 2 shows the marginal stability curves given by Pearson in the case of fixed conducting liquid/solid boundary (1958). Pearson's results were given by the curves for $L=0,2,4,6$, and the curve $L=1$ can be evaluated from the curves $L=0$ and $L=2$, as shown by the dashed line in Fig. 2. It is obvious that the values of $B$ and $\alpha$ calculated by the present code agrees well with Pearson's result, which provides a good validation for the code.

\section{Numerical results}

Fig. 3 shows the stream lines and isotherms of the liquid layer with non-deformed free surface. The side walls are adiabatic and impermeable but slippery. The volume ratio $V / V_{0}=1$, where $V$ is the practical volume of liquid layer and $V_{0}$ is the volume of liquid $1.5^{\circ} \mathrm{C}$

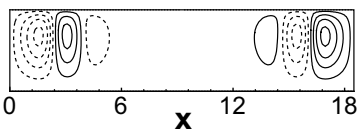

$1.5^{\circ} \mathrm{C}$

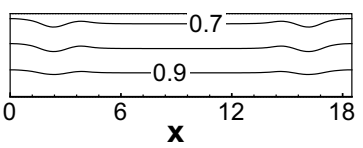

$1.8^{\circ} \mathrm{C}$
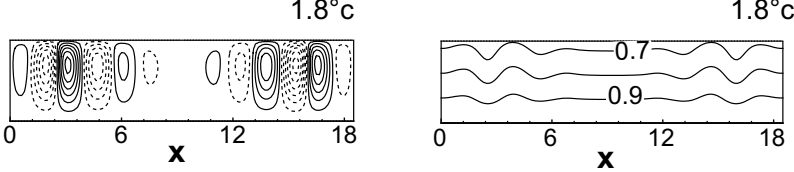

$1.97^{\circ} \mathrm{C}$
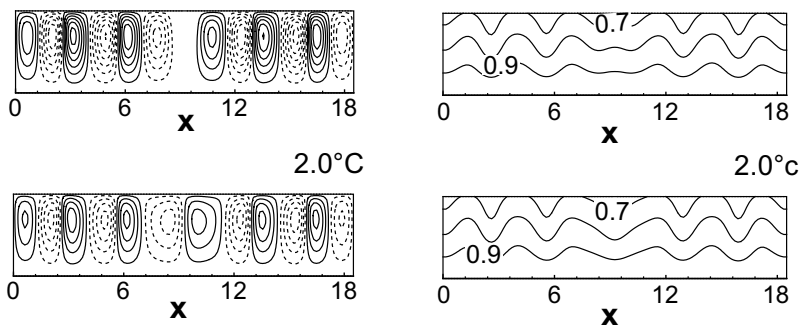

$2.2^{\circ} \mathrm{C}$
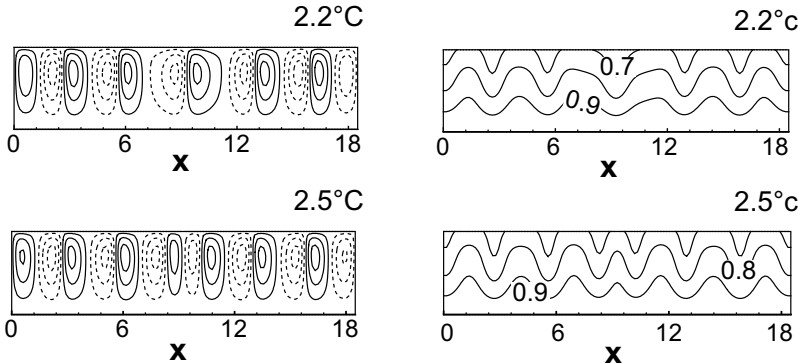

Fig. 3. Streamline (left), isotherms (right) in planer liquid layer, $V / V_{0}=1$.

layer with planar free surface. In Fig. 3, the applied temperature difference $\Delta T$ changes from $1.5^{\circ} \mathrm{C}$ to $2.5^{\circ} \mathrm{C}$. A pair of rolls appears at first near the side walls and then the roll pairs evolved one by one when the temperature difference $\Delta T$ is increased.

The maximum dimensionless vertical velocity $W_{\mathrm{c}}$ at central line of the liquid layer with different volume ratio is shown in Fig. 4, where $W_{c}=W_{0} h / v, W_{0}$ is the dimensional velocity and $v$ is the kinematic viscosity of liquid. The solid dots in the Fig. 4 correspond to the critical points of emerging Marangoni convection for liquid layers of different volume-ratios. It is shown that the maximum upward (or downward) velocity $W_{c}$ at a given line is nearly zero if the temperature difference is lower than the critical value $\Delta T_{\mathrm{C}}$, otherwise it increases sharply. It is obvious that the critical temperature difference (critical Marangoni number) of emerging 


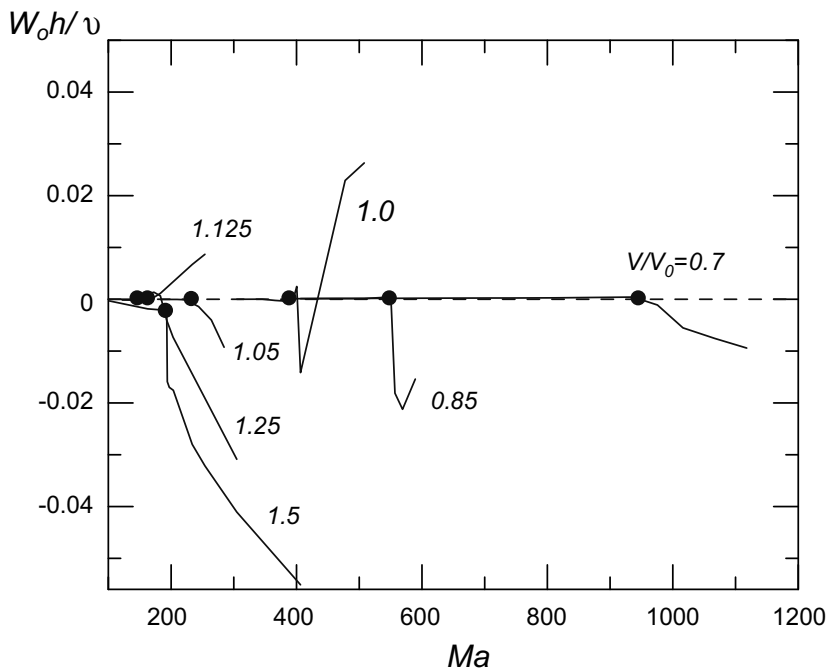

Fig. 4. The maximum dimensionless vertical velocity $W_{c}$ and related Marangoni number at the symmetric axis of the liquid layer with volume ratio $0.7-1.5$.

Marangoni convection depends on the volume ratio of the liquid layer. The larger the volume ratio, the lower the critical temperature difference (or critical Marangoni number).

We need to discuss how to determine the critical temperature difference or critical Ma number for the onset of Marangoni convection in the liquid layer of finite extension with a convex or a concave free surface. In case of infinite liquid layer heated from below, Marangoni convection appears when a critical value of the Marangoni number $\left(M a_{c}\right)$ is exceeded, and regular flow rolls arise suddenly in the whole liquid layer. The upward velocity $W$ at the place between two rolls becomes large obviously. In the planar liquid layer of finite extension, due to the effect of the side walls a pair of convective roll emerges at first in the region near the side walls, even the boundaries are slippery which means zero shear stress applied on the boundary. The number of convective rolls gradually increases and the rolls gradually fill up the liquid layer with the increase of the temperature difference. The critical state is defined at the moment when Marangoni rolls appear at both sides of the symmetric axis in the liquid layer, and the related temperature difference is called critical temperature difference $\Delta T_{\mathrm{c}}$. The central upward velocity increases suddenly at the critical state, therefore, the $z$-direction velocity $W$ is used as a criterion to judge quantitatively whether the Marangoni convection is excited. Furthermore, the velocity $W$ changes between positive value and negative value alternatively although it is nearly zero in the subcritical state. When $W$ changes from negative (positive) to positive (negative) and then increases sharply, the applied temperature difference is defined as $\Delta T_{\mathrm{c}}$ in the planar liquid layer of finite extension. For present case, the critical temperature difference is $1.93{ }^{\circ} \mathrm{C}$, and related Marangoni number equals 392 . There are six pair rolls in the whole liquid layer in the critical state. Then the strength of the rolls and rolls number increase with increasing $\Delta T$.

The streamline patterns and isotherms of the liquid layer with convex free surface $\left(V / V_{0}=1.5\right)$ are shown in Fig. 5. Two cells appear in the whole region in the sub-critical state induced by the thermocapillary convection driven by the surface tension gradient, and new pair of cells grows and splits from the original pair gradually. When the maximum normal velocity $W$ at the symmetric axis of the liquid layer changes from negative to positive, the $\Delta T$ is defined as $\Delta T_{\mathrm{c}}$. For present case, $\Delta T_{\mathrm{c}}=0.955^{\circ} \mathrm{C}$ and $M a_{\mathrm{c}}=194$. Three pair rolls appear in the critical state. Fig. 6 shows that the cells appear near the walls at first, and then evolve towards the symmetric axis of the liquid layer with concave free surface
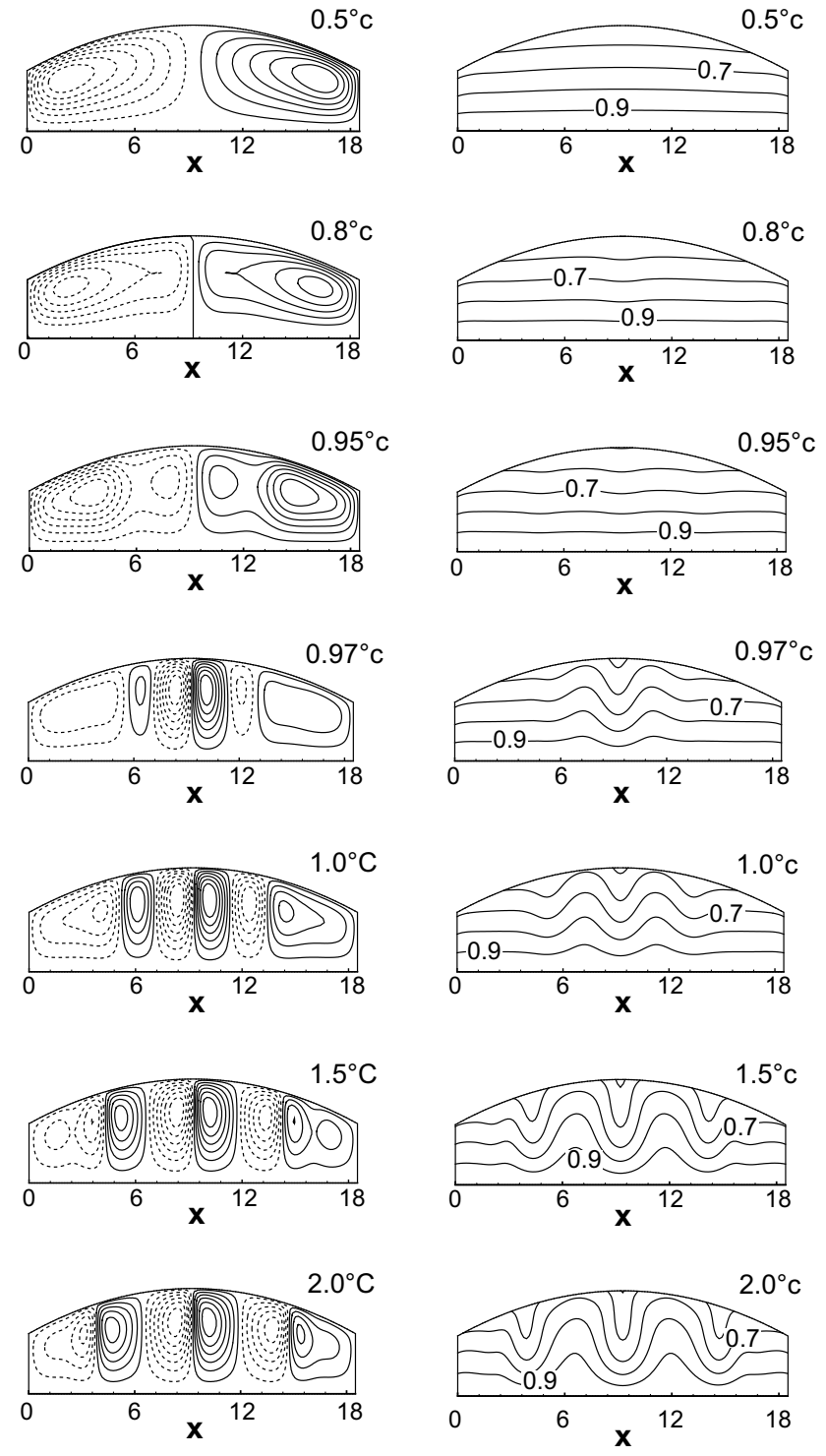

Fig. 5. Streamline (left), isotherms (right) in convex liquid layer, $V / V_{0}=1.5$.

$\left(V / V_{0}=0.7\right)$. When the maximum normal velocity $W$ at the symmetric axis of the liquid layer changes from positive to negative, the $\Delta T$ is defined as $\Delta T_{\mathrm{c}}$. Here, $\Delta T_{\mathrm{c}}=4.66^{\circ} \mathrm{C}$ and related $M a_{\mathrm{c}}=946.7$. Nine pair rolls appear in the critical state.

The critical temperature difference $\Delta T_{\mathrm{c}}$ and critical Marangoni number $M a_{\mathrm{c}}$ change with the volume ratio $V / V_{0}$ (Fig. $7 \mathrm{a}$ and $\mathrm{b}$ ). The related curve is divided into two branches. The left branch corresponds to $V / V_{0}<1$, and the right one corresponds to $V / V_{0}>1$. The curved free surface introduces the non-uniform temperature distribution on the free surface, and then induces the thermocapillary flow. Correspondingly, the bifurcation structure and critical temperature difference are changed. There is a bifurcation sequence depending on the volume ratio. The rolls appear firstly near the side wall and then evolve towards the symmetric axis in the planar and concave liquid layer. In case of concaved liquid layer of $V / V_{0}=0.7$, there are 9 pairs of rolls in the critical state and only 6 pairs rolls in case of the planar liquid layer. There is a pair roll appears first in the whole liquid region due to the thermocapillary convection and new cells are grow gradually from the original cells and split to form new pair in convex liquid layer with $V / V_{0}=1.5$. There are three pairs of rolls in the critical state. The different bifurcation structure and 

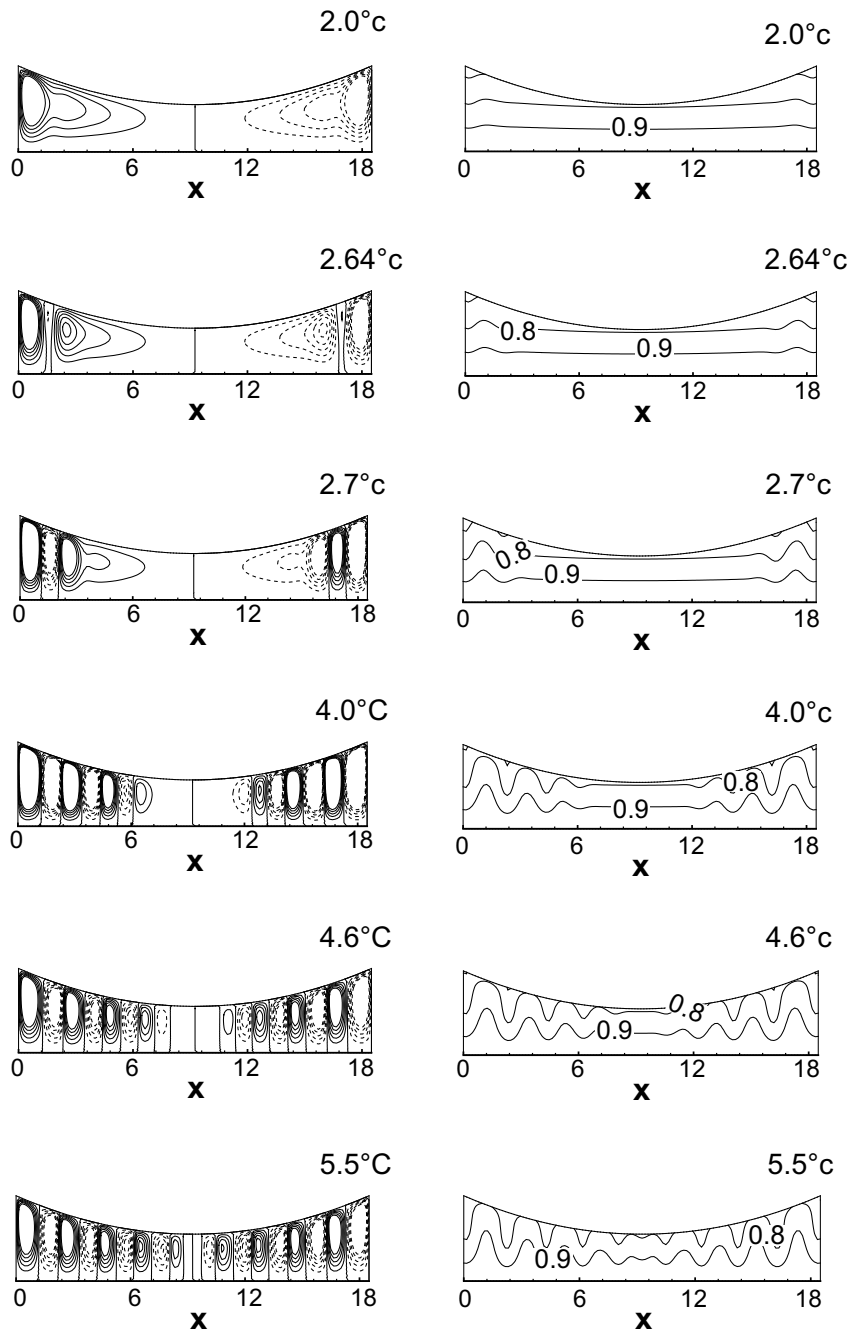

Fig. 6. Streamline (left), isotherms (right) in concave liquid layer, $V / V_{0}=0.7$.

critical temperature difference for liquid layers of different volume ratios are due to two reasons. One reason is the different configuration of the liquid layer. The height of the concave layer is lower than $h_{0}$, therefore, the corresponding critical temperature difference is higher than that of the planar liquid layer, and the number of the roll pairs is increased. The height of the convex liquid layer is higher than $h_{0}$, so the corresponding critical temperature difference is lower than that of the planar liquid layer, and the number of the roll pairs is decreased. The other reason is the thermocapillary convection in the concave and convex liquid layers. For the concave one, the minimum height of liquid layer and the maximum temperature at the free surface are at the symmetric axis. The thermocapillary flow is from the symmetric axis to the side walls at the free surface. For the convex one, the situation is opposite. The thermocapillary convection will influence the critical temperature difference or critical Marangoni number. However, the main influence on the critical temperature difference is from the liquid layer configuration. Therefore, the relationship curve in Fig. 7 is divided two branches, which shows that the larger the volume ratio, the lower the critical temperature difference.

\section{Discussion}

In the present paper, the influence of the volume ratio of a liquid layer on the Pearson's cells was studied in a two-dimensional
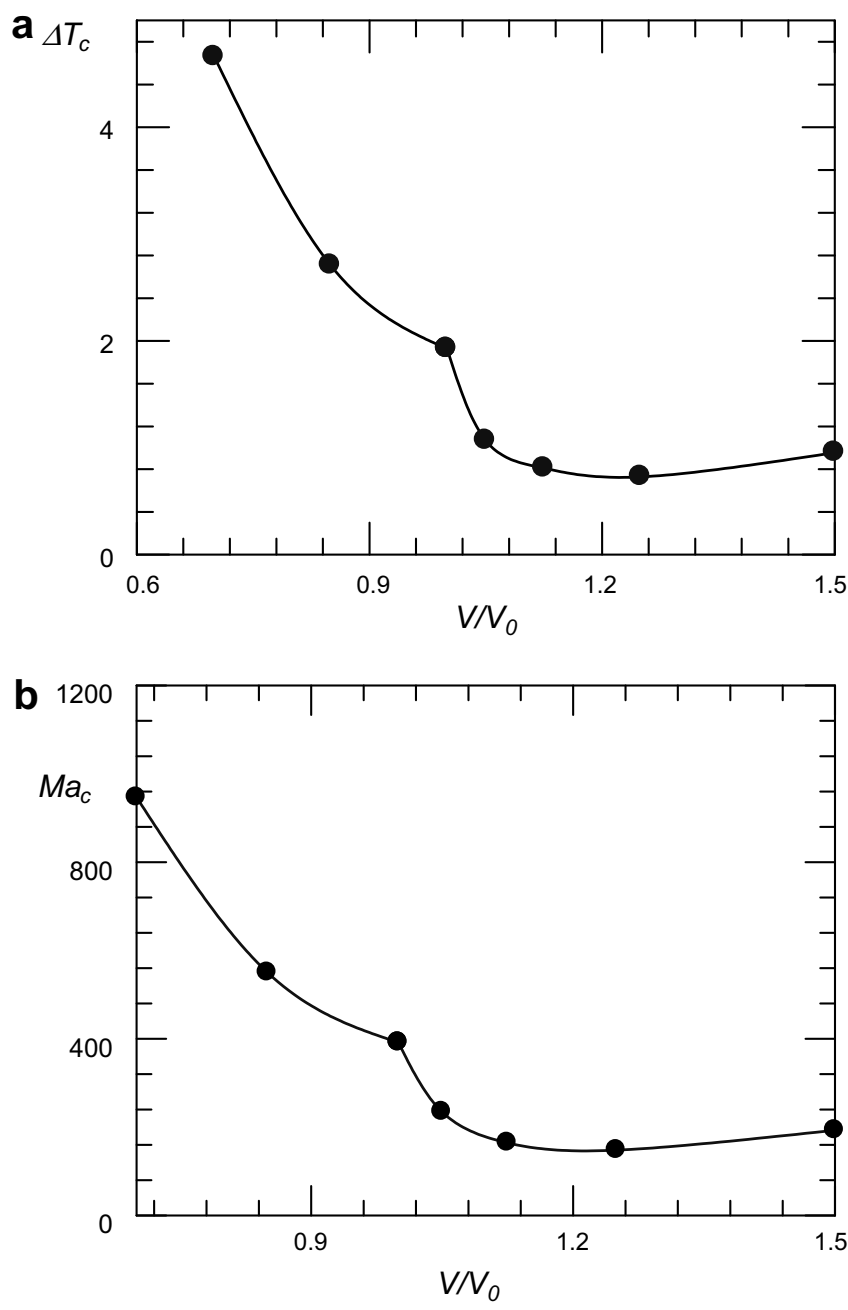

Fig. 7. (a) The relationship of critical temperature difference and volume ratio $V / V_{0}$; and (b) the relationship of critical Marangoni number and volume ratio $v / v_{0}$.

numerical simulation model. The relationship curve of the critical applied temperature difference or critical Ma number and volume ratio consists two branches separated by $V / V_{0}=1$, i.e. $V / V_{0}>1$ and $V / V_{0}<1$, due to the configuration of liquid layer and thermocapillary convection. However, the main influence is from the former. The numerical results also revealed that a pair of cells appears at first in the whole region or near the lateral walls depending on the system configuration, and then new pairs of cells evolve gradually to cover the whole region with the increasing temperature difference. These phenomena are quite different from the linear stability analysis results, in which a series of regular flow cells appears at the critical temperature difference.

It is worthy noted that in the linear stability analyses, the marginal curves can be obtained by solving an eigenvalue problem involving the parameters of $M a, L$, and the critical conditions are independent of $\operatorname{Pr}$. However, the critical Ma number and the convective structure will definitely be influenced by $\operatorname{Pr}$ number in the practical problem. The influence of $\operatorname{Pr}$ number on the Pearson's cells is necessary to be studied by the numerical simulations and experiments in the future.

\section{Acknowledgement}

This research is supported by the National Natural Science Foundation of China (No. 10432060). 


\section{References}

[1] J.R.A. Pearson, On convection cells induced by surface tension, J. Fluid Mech. 4 (1958) 489-500.

[2] L. Rayleigh, Scientific Papers, vol. 6, Cambridge University Press, 1916. p. 432

[3] S. Rosenblat, S.H. Davis, G.M. Homsy, Nonlinear Marangoni convection in bounded layers. Part 1. Circular cylindrical containers, J. Fluid Mech. 120 (1982) 91-122.

[4] S. Rosenblat, G.M. Homsy, S.H. Davis, Nonlinear Marangoni convection in bounded layers. Part 2. Rectangular cylindrical containers, J. Fluid Mech. 120 (1982) 123-138.

[5] H.A. Dijkstra, On the structure of cellular patterns in Rayleigh-BenardMarangoni flows in two-dimensional containers with rigid sidewalls, J. Fluid Mech. 243 (1992) 73-102.

[6] H.A. Dijkstra, Surface tension driven cellular patterns in three-dimensional boxesPart II: a bifurcation study, Microgravity Sci. Technol. VIII (2) (1995) 70-76.
[7] M.F. Schatz, G.P. Neitzel, Experiments on thermocapillary instability, Ann. Rev. Fluid Mech. 33 (2001) 93-127.

[8] A. Alexander, G.-R. Tatiana, S. Perter, Marangoni convection and heat transfer in thin liquid films on heated walls with topography: experiments and numerical study, Phys. Fluid 17 (2005) 062106106210613.

[9] E.S. Oleg, A.N. Alexander, Thermocapillary flows under an inclined temperature gradient, J. Fluid Mech. 504 (2004) 99-132.

[10] N.N. Vanenko, The Method of Fractional Steps, Springer-Verlag, Berlin, Heidellerg, New York, 1971.

[11] J.H. Wu, A hybrid method of fractional steps with L-stability for numerical modeling of aquatic environments, Adv. Hydrodynam. 1 (3) (1985) 27-36 (in Chinese).

[12] B.M. Carpenter, G.M. Homsy, High Marangoni number convection in a square cavity: Part II, Phys. Fluid A2 (2) (1990) 137-149. 